\title{
La expresión de los eventos de colocación en danés y español
}

\author{
Iraide Ibarretxe-Antuñano* \\ Teresa Cadierno** \\ Alberto Hijazo-Gascón***
}

\begin{abstract}
Resumo
Los eventos de colocación (placement events) en los que una Figura cambia de posición por la acción de un agente (ej. Pone una taza en la mesa) suponen un interesante ámbito de investigación para la Lingüística Cognitiva, en especial para los estudios relacionados con cuestiones neorrelativistas. Este artículo analiza en primer lugar la codificación de los eventos de colocación en dos lenguas tipológicamente distintas (TALMY, 1991, 2000): el danés (marco satélite, CADIERNO, 2004) y el español (marco verbal, TALMY, 1991; SEBASTIÁN; SLOBIN, 1994). En segundo lugar, a partir de los resultados obtenidos en la descripción interlingüística, se formulan algunas hipótesis sobre las estrategias y dificultades que los aprendientes nativos daneses de español como segunda lengua se pueden encontrar a la hora de hablar sobre este tipo de eventos desde la perspectiva del Pensar para Hablar (CADIERNO, 2008; SLOBIN, 1991). Los datos se han obtenido a partir de los estímulos desarrollados dentro de PUT-project del Instituto Max Planck de Psicolingüística de Nimega (Países Bajos) (BOWERMAN et al., 2004; KOPECKA; NARASIMHAN, 2012).

Palabras clave: Eventos de colocación. Español. Danés. Variación intertipológica. Pensar para hablar.
\end{abstract}

\section{Los eventos de colocación}

Un evento de colocación se puede definir como el evento en el que una Figura cambia de posición por la acción de un agente. Este tipo de eventos se han denominado por autores como Talmy $(1985,2000)$ 'eventos de movimiento causado'. Estos eventos se pueden describir a través de diversos componentes *Universidad de Zaragoza.

**University of Southern Denmark.

***University of East Anglia. Esta investigación se enmarca dentro del proyecto de investigación MovEs financiado por el Ministerio de Economía y Competitividad del Gobierno de España (FFI201014903, FFI2013-45553-C3-1-P), así como por la Fundación Velux de Dinamarca. Nos gustaría dar las gracias a Moiken Jessen e Isabel Casas por su ayuda con los análisis estadísticos. 
semánticos que representan las piezas de información semántica fundamentales que constituyen y hacen que un evento se considere de colocación. Estos elementos son: el Agente que hace posible el cambio de posición, la Figura que es el elemento que cambia de posición, el Camino que sería la trayectoria o la posición que tiene la Figura, y la Base que representaría el nuevo lugar donde se coloca la Figura. Por ejemplo, en el enunciado La mujer pone una taza en la mesa, el Agente sería la mujer, la Figura sería una taza, el Camino sería en y, finalmente, la Figura sería la mesa.

El estudio de la codificación de los eventos de colocación es interesante no solo por el aporte general que puede ofrecer para confirmar (o desechar) teorías como la de los patrones de lexicalización de Talmy $(1991 ; 2000)$ que veremos en la siguiente sección, sino también desde el punto de vista del neorrelativismo lingüístico y de las posibles consecuencias de este en la adquisición de segundas lenguas. Los aprendientes tienen que adaptarse al estilo retórico de la lengua meta: esto no solamente consiste en generar frases gramaticalmente correctas sino también enunciados que sean parecidos a los que los hablantes nativos de la lengua producen en situaciones discursivas similares. En otras palabras, los aprendientes tienen que ser conscientes de las similitudes y diferencias que existen entre su lengua nativa y su(s) segunda(s) lengua(s) a la hora de codificar el evento de codificación, y tratar de adaptarse y ajustar sus patrones nativos a las características léxicas y sintácticas de la segunda lengua. Este fenómeno se ha denominado 'aprender a pensar en una segunda lengua' (CADIERNO, 2004, 2008, 2010) o 'aprender a re-pensar para hablar' (ROBINSON; ELLIS, 2008).

En este artículo analizamos los eventos de colocación en dos lenguas tipológicamente distintas (TALMY, 1991, 2000): el danés (marco satélite, CADIERNO, 2004) y el español (marco verbal, TALMY, 1991; SEBASTIÁN; SLOBIN, 1994). Tomando como punto de partida los experimentos diseñados por el PUT-Project del Instituto Max Planck de Psicolingüística de Nimega (Países Bajos) (BOWERMAN et al., 2004; KOPECKA; NARASIMHAN, 2012), este articulo examina en primer lugar la expresión de los eventos de colocación (PONER) por hablantes nativos de danés y español. Se demuestra cómo los hablantes de danés prestan atención a la posición de la Figura (stille 'poner verticalmente' vs. laegge 'poner horizontalmente'), mientras que los hablantes de español se fijan más en los diferentes grados de dinámica de fuerzas e intencionalidad de los eventos (caerse $>$ caérsele $>$ dejar caer $>$ tirar, IBARRETXE-ANTUÑANO, 2012). En segundo 
lugar, y partiendo de la teoría del pensar para hablar de Slobin (1991), se presentan algunas hipótesis sobre las posibles dificultades que los aprendientes daneses de español como segunda lengua puedan encontrarse a la hora de adaptarse al estilo retórico de la lengua meta y de las estrategias de comunicación que éstos puedan adoptar para describir el evento de colocación.

\section{La expresión de eventos de colocación en dos lenguas tipológicamente diferentes}

En su teoría de los patrones de lexicalización, Talmy $(1991,2000)$ divide a las lenguas del mundo en dos grandes grupos: las lenguas de marco verbal, que son aquellas en las que el componente semántico central de un evento se codifica en el verbo principal, y las lenguas de marco de satélite, en las que este componente central aparece en un satélite, es decir, en un elemento fuera del verbo sin relación de hermandad. El campo en el que más se ha aplicado la tipología de Talmy es el del movimiento. Así, las diferencias entre las lenguas de marco verbal y de marco de satélite se pueden ver ilustradas en los ejemplos La botella salió flotando y The bottle floated out 'la botella flotó fuera' en los que el componente principal del movimiento, el Camino o trayectoria, se encuentra en el verbo principal salir para el español y en el satélite out en inglés. Partiendo de esta distinción tipológica, Slobin (1991, 1996, 2000, 2004, 2006) propone la hipótesis del Pensar para Hablar. Esta hipótesis postula que los hablantes de una lengua se ven influenciados por las herramientas lingüísticas que las lenguas ponen a su disposición. En otras palabras, los hablantes han de describir un evento utilizando obligatoriamente los mecanismos lingüísticos de su lengua. Es por este motivo que los hablantes de una determinada lengua tienen su propio 'estilo retórico', su forma característica de describir y codificar una determinada situación. Este estilo retórico no es necesariamente gramatical, ${ }^{1}$ es decir, el hablante no se ve obligado a utilizar unas determinadas estructuras por una razón de gramaticalidad, sino que simplemente de entre todas las posibilidades que tiene a su disposición hay una serie de estructuras que aparecen de forma más prominente para describir un determinado evento.

1 Esto no quiere decir que los distintos estilos retóricos no puedan resultar de las diferentes categorías gramaticales obligatorias que existen en diversas lenguas, pero no es un requisito indispensable. 
La mayoría de las investigaciones sobre el Pensar para Hablar se centran en eventos de movimiento intransitivo o movimiento espontáneo. Sin embargo, estudios como los recogidos en Kopecka y Narasimhan (2012) han demostrado que el movimiento causado o los eventos de colocación son también de interés desde la perspectiva de la variación interlingüística. Por un lado, desde el punto de vista de la semántica léxica, ya que es interesante conocer qué verbos se usan para distinguir entre diferentes tipos de eventos de colocación. Por otro lado, desde el punto de vista de las correspondencias entre la sintaxis y la semántica, es decir, cómo se distribuyen las nociones semánticas espaciales a través de los diversos elementos que componen estas construcciones tanto a nivel oracional como a nivel sintagmático.

Este trabajo se centra en el primer aspecto, es decir, en el estudio de la semántica léxica de los verbos utilizados por los hablantes nativos para distinguir diferentes tipos de eventos de colocación en danés y español. En concreto, este trabajo se plantea las siguientes preguntas de investigación:

- Pregunta 1: ¿Qué verbos utilizan los hablantes nativos de danés y español para describir eventos de colocación? ¿Existen diferencias intertipológicas?

- Pregunta 2: ¿Cuáles son los significados de estos verbos? Es decir, ¿qué categorías semánticas cubren / reflejan? ¿Son comparables las categorías de los verbos daneses y las de los verbos españoles?

- Pregunta 3: ¿Qué dificultades se pueden encontrar los aprendientes nativos daneses a la hora de utilizar los verbos de colocación en español como L2?

\section{Metodología}

\subsection{Participantes}

El estudio se ha llevado a cabo con 14 hablantes nativos de danés y con 10 hablantes nativos de español (los resultados provienen de IBARRETXEANTUÑANO, 2012). La recogida de datos se llevó a cabo en la Universidad del Sur de Dinamarca (Odense, Dinamarca) y en la Universidad de Zaragoza (España), respectivamente. 


\subsection{Materiales}

Para la obtención de datos se ha utilizado la tarea conocida como PUT task desarrollada en el Instituto de Psicolingüística del Max Planck (Nimega, Holanda) (BOWERMAN et al., 2004; KOPECKA; NARASIMHAN, 2012). Esta tarea consta de 63 video-clips de corta duración (3-4 segundos) en los que se realizan diferentes tipos de eventos de colocación, tanto de PONER como de QUITAR. Dado el objetivo de investigación intertipológica de estos materiales, los estímulos de los videos atienden a diferentes aspectos que pueden intervenir en la conceptualización de este tipo de eventos por hablantes de diversas lenguas, tales como el tipo de Figura (rígida-flexible, granular-líquida, forma, etc.) y de Base (animada-inanimada, horizontal-vertical, superficie-apoyo), la configuración espacial entre la Figura y la Base (contenido-apoyado, encaje amplio-ajustado, etc.), tipos de instrumento con que se llevan a cabo las acciones de colocación (mano u otra parte del cuerpo del agente, pinzas, etc.) o la manera en la que se llevan a cabo (deliberado-accidental, etc.). En la Figura 1, se muestras tres ejemplos de los videos que aparecen en esta tarea (véase BOWERMAN et al., 2004, y NARASIMHAN et al., 2012 para una descripción más detallada).
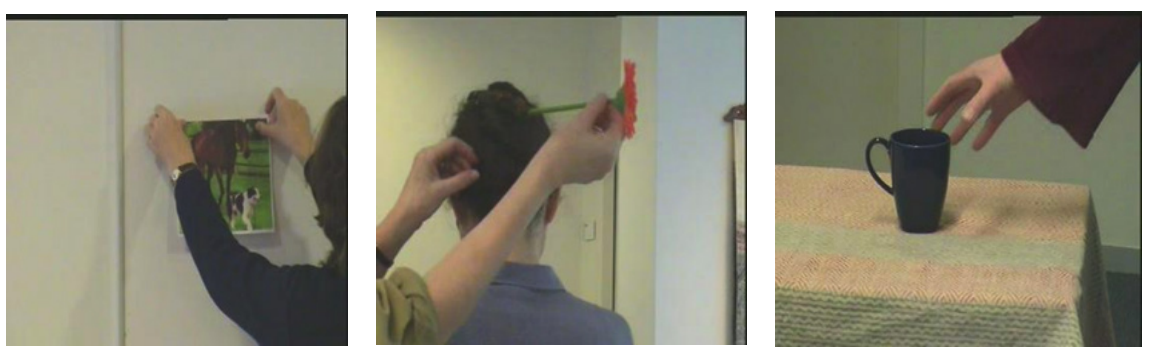

Figura 1: Ejemplos del PUT task (BOWERMAN et ál. 2004).

Para este artículo, se han seleccionado exclusivamente los vídeos que representan acciones de PONER (utilizamos la versalita para señalar la categoría), es decir, un total de 31 vídeos. 


\subsection{Procedimiento}

Los informantes, en sesiones individuales, veían cada video una vez en un orden determinado en el ordenador. Su tarea consistía en describir oralmente lo que ocurría en cada uno de los videos. Para evitar cualquier efecto en la elicitación de estos datos, los videos están organizados en tres grupos ordenados aleatoriamente. Cada sesión se grabó en grabadora/vídeo para su transcripción posterior.

\section{Resultados}

\subsection{Tipos y ocurrencias de los verbos de colocación (PONER)}

El primer resultado tiene que ver con el tipo de verbos de PONER y el número de ocurrencias de estos verbos en los hablantes nativos de español y danés. Los resultados se resumen en la Tabla 1.

Tabla 1: Valores de media para ocurrencias y tipos de verbos de colocación (PONER)

\begin{tabular}{|c|c|c|c|}
\hline & $\begin{array}{c}\text { Tipos } \\
\text { (types) }\end{array}$ & $\begin{array}{c}\text { Ocurrencias } \\
\text { (tokens) }\end{array}$ & $\begin{array}{c}\text { Ratio } \\
\text { (type-token } \\
\text { ratio) }\end{array}$ \\
\hline Español & 12.5 & 30.9 & 0.40 \\
\hline Danés & 12.86 & 31 & 0.41 \\
\hline
\end{tabular}

Además del valor medio para los tipos y las ocurrencias de los verbos de PONER en estos hablantes, la Tabla 1 también recoge el cálculo de la ratio entre el tipo y la ocurrencia que examina los tipos de verbos usados en relación al número medio de las ocurrencias de los verbos obtenidos. Esta ratio es importante porque nos aporta una descripción de los datos más equilibrada ya que el número de ocurrencias varía en función del grupo lingüístico.

Después de llevar a cabo el test U de Mann-Whitney sobre el ratio entre el tipo y la ocurrencia, no se han encontrado diferencias significativas entre los hablantes de español y de danés $(\mathrm{p}=0.584)$. En definitiva, lo que muestra la Tabla 1 es que 
el número de tipos de verbos que los hablantes de danés y de español utilizan para describir estos vídeos es muy similar a pesar de que pertenecen a patrones de lexicalización diferentes (las lenguas de marco satélite suelen tener un léxico más detallado y numeroso). Una vez que hemos examinado la primera pregunta de investigación que nos planteábamos en la sección 2 , el siguiente paso será ver cuáles de estos verbos son los que se utilizan con más frecuencia en cada grupo.

\subsection{Tipos y frecuencia de uso de verbos de colocación (PONER)}

A pesar de que el número de tipos de verbos en ambas lenguas es relativamente similar, si se analiza en detalle el tipo de información que estos verbos codifican en estas lenguas se pueden apreciar algunas semejanzas pero también algunas diferencias importantes. Los verbos utilizados en español y en danés están resumidos en (1) y (2).

(1) Verbos de colocación en español

Arrojar, caerse, colgar, colocar, colocarse, dar, dejar, dejar caer, depositar, derramar, derramarse, echar, guardar, guardarse, introducir, lanzar, meter, meterse, pasar, pegar, poner, ponerse, posar, tapar, taponar, tirar, verter

(2) Verbos de colocación en danés

aflevere 'entregar', balancere 'balancearse', falde 'caer(se)', flyde (over) 'fluir, desbordar', give 'dar', aholde 'verter', hoenge 'colgar', kaste 'tirar, lanzar', komme 'venir', lade falde 'dejar caer', lagge 'poner / meter horizontalmente', aplacere 'colocar, poner', proppe 'meter, taponar', putte 'poner, meter', rcekke 'pasar, dar', rule 'rodar', ryge 'precipitarse', scette 'poner verticalmente', skubbe 'empujar', smide 'tirar, echar, lanzar', spilde 'derramar, verter', stikke 'meter, picar, punzar, pinchar', stille 'poner, colocar verticalmente', stoppe 'rellenar', tabe 'perder, dejar caer', tage 'tomar, coger', tippe 'bascular, volcar', trcekke 'tirar (hacia sí mismo)', vippe 'balancear(se)', vrikke 'menearse, contonearse'

Los verbos de colocación en danés y en español incluyen casos en los que se reflejan las mismas nociones semánticas (contenedor, meter y putte) y casos en los que las diferencias semánticas no se ven reflejadas en una de las dos lenguas (la posición vertical u horizontal del danés—stille vs. laggge-ausente en español). 
Sin embargo, el hecho de que haya diferentes tipos no significa que los hablantes utilicen todos estos tipos con la misma frecuencia para describir los vídeos del estudio. Es posible que algunos verbos sean más frecuentes que otros. La Figura 2 recoge la frecuencia de uso de los verbos en español.

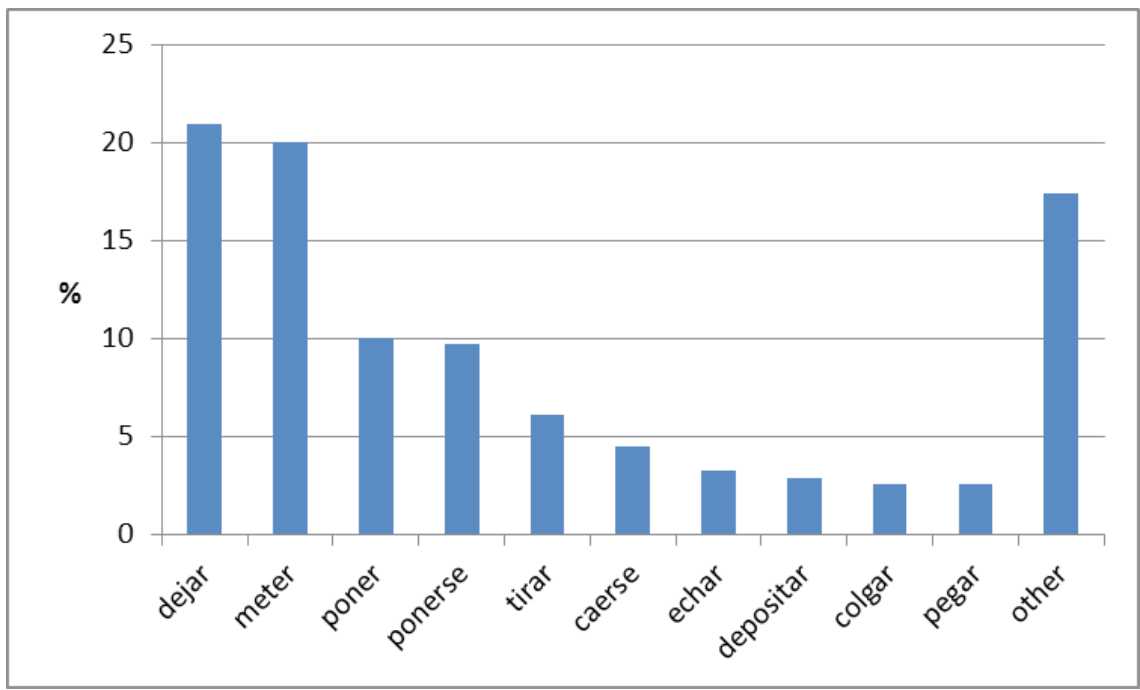

Figura 2: verbos de colocación (PONER) en español L1

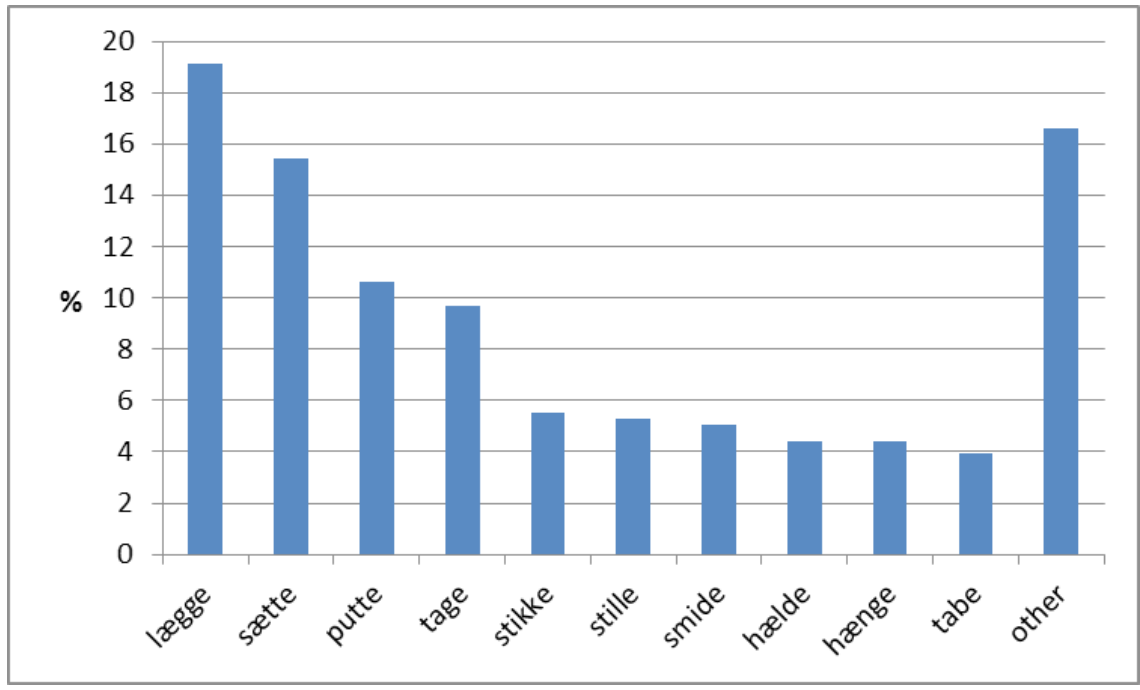

Figura 3: verbos de colocación (PONER) en danés L1 
Los hablantes nativos de español utilizan predominantemente dos verbos de colocación, dejar - que refleja la intencionalidad del hablante-y meterque codifica la trayectoria exterior-interior, seguidos después por el verbo más neutral, poner. Los hablantes nativos de danés siguen una tendencia muy parecida a los de español como se observa en la Figura 3. Hay dos verbos que se utilizan preferentemente, lagge 'poner horizontalmente' y satte 'poner verticalmente', seguidos del verbo putte 'poner, meter'. Es interesante destacar dos apreciaciones. Por un lado, los verbos más usados reflejan directamente algunas de las diferencias semánticas que hemos destacado para ambas lenguas como la necesidad de especificar la verticalidad/horizontalidad de la Figura en danés ausente en español. Por otro lado, la frecuencia de uso de estos verbos en las dos lenguas se puede considerar como un continuo; en ambas destaca un grupo reducido de verbos muy frecuentes, al que le sigue un grupo de uso medio (Esp. caerse, echar; Dan. stille 'poner verticalmente', hcelde 'verter'), para terminar con una categoría que hemos denominado 'otros' y que recoge verbos que se usan esporádicamente (Esp. colocar(se), posar, verter, derramar, taponar, tapar, introducir, lanzar, arrojar y dejar caer; Dan., balancere 'balancear', vrikke 'menear', rulle 'rodar', skubbe 'empujar', tippe 'volcar', kaste 'tirar', lade falde 'dejar caer').

\subsection{Distribución de los tipos de verbos por escenas: categorización semántica}

Una vez que hemos analizado el tipo y las ocurrencias de los verbos de colocación (PONER) en estos videos en general, el siguiente paso es preguntarse por los significados de los verbos que se utilizan en estos videos. En otras palabras, necesitamos saber cuáles son las categorías semánticas que cubren o que se reflejan en los verbos empleados para saber si los hablantes de danés y español coindicen o no en su manera de agrupar los eventos de colocación.

Para poder responder a esta pregunta hemos utilizado el análisis de cluster o de conglomerados (véase MAJID, 2012). Esta es una técnica que nos permite agrupar en un mismo cluster o conglomerado los vídeos que se describen con los mismos verbos. Cuanto más homogénea sea la descripción de las escenas en relación al tipo de verbo utilizado, más pequeña será la distancia que separa los clusters que se forman (las cajas) de la línea horizontal. Este tipo de análisis 
ya ha sido utilizado anteriormente para estudiar la categorización semántica de otros tipos de eventos como los de cortar y romper (véase MAJID et al., 2007).

En la Figura 4, se pueden ver los diferentes conglomerados que han surgido de los datos de español. El primer cluster corresponde al verbo dejar y agrupa escenas que describen una relación de soporte entre la Figura y la Base (PUT CUP ON TABLE). El segundo cluster se agrupa en torno al verbo ponerse e incluye escenas de eventos de ponerse ropa, es decir, escenas en las que la Figura se refiere a un tipo de ropa y la Base es una parte del cuerpo del agente. El tercer cluster pertenece al verbo poner y recoge tanto escenas de soporte (PUT SAUCER ON TOP OF CUP) como escenas de relaciones de inclusión parcial (PUT A CANDLE INTO A CANDLE STAND / PUT FLOWER INTO HAIR). El verbo tirar conforma el cuarto cluster que describe escenas en las que hay diferentes acciones relacionadas con el Agente que se desprende de la Figura que termina en la Base (suelo) con distintos grados de fuerza e intencionalidad (TOSS BOOK ON FLOOR VS. DROP BOOK DELIBERATELY ONTO FLOOR). El quinto cluster corresponde al verbo caerse que recoge escenas en las que la Figura, como en el cluster anterior, también termina en el suelo, pero con una diferencia muy importante: hay una falta absoluta de intencionalidad que se ve reflejada precisamente en el uso pronominal del verbo caerse. El sexto cluster se congrega alrededor del verbo meter que se refiere a escenas en las que existe una relación espacial de inclusión total (PUT HEAD INTO A BUCKET). El uso de un verbo como meter que tiene codificado el componente de Camino es típico de lenguas de marco verbal como el español. Finalmente, hay un último cluster, marcado con la línea de puntos discontinua, en el que no hay un verbo predominante para describir cada escena sino varios. La elección de estos verbos depende de varios factores. Por ejemplo, en las escenas en las que la Figura es líquida o granular (POUR LIQUID INTO CONTAINER), se usan verbos cuya semántica recoge este tipo de información, como verter o derramar. En otras escenas en las que se describe la Manera en la que se coloca algo se utiliza un verbo específico como colgar (HANG ROPE OVER TREE BRANCH). El uso de estos verbos tan específicos plantea cuestiones interesantes relacionadas con los patrones de lexicalización de Talmy que deberán estudiarse con más detalle en el futuro, como por ejemplo, el hecho de que existen verbos que codifican características de la Figura (verter) o que la Manera también aparezca específicamente en el verbo principal. 


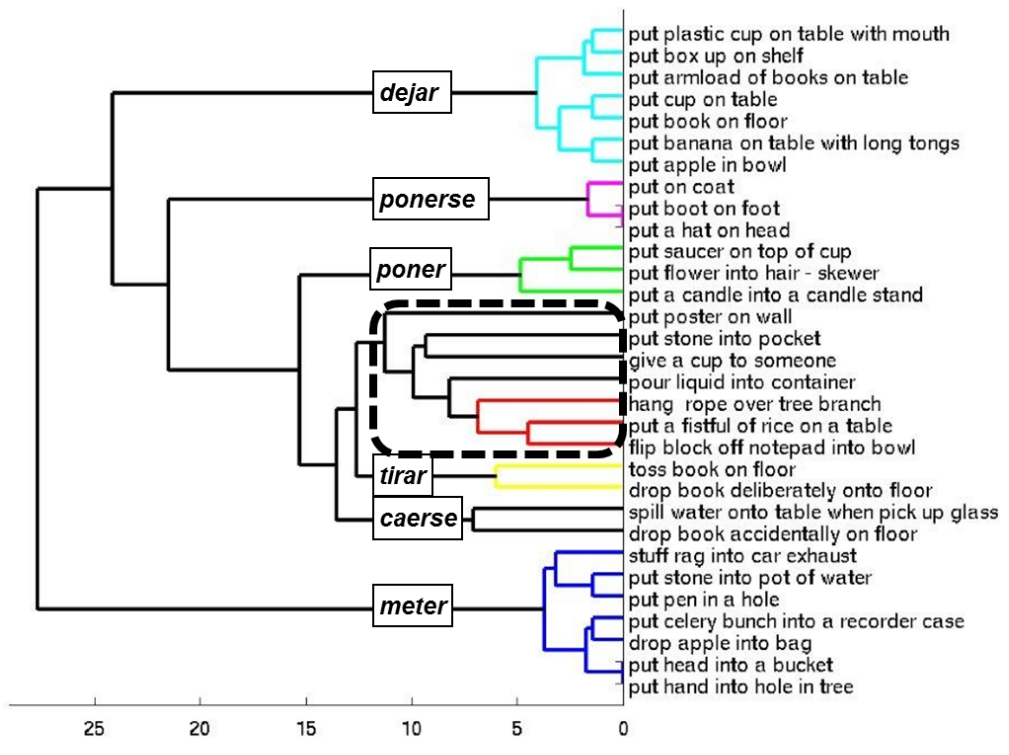

Figura 4: Dendograma para L1 español

En la Figura 5 se ve el dendograma correspondiente a los datos del danés. El primer cluster se agrupa en torno al verbo laegge 'poner horizontalmente' y corresponde a escenas que describen una relación de contenedor (PUT PEN IN A whOLE) y de soporte entre la Figura y la Base (PUT BOOK ON FLOOR) pero en las que la Figura se encuentra en posición horizontal. El segundo cluster se describe principalmente con los verbos satte y stille 'poner verticalmente'; en este caso existe una relación de soporte pero en estos casos hay una relación de verticalidad entre la Figura y la Base. El tercer cluster corresponde al verbo scette 'poner' exclusivamente. Este verbo se utiliza para cubrir escenas en las que existe una relación de sorporte (PUT PLASTIC CUP ON TABLE WITH MOUTH) pero también en las que existe una relación de inclusión parcial (PUT FLOWER INTO HAIR, PUT A CANDLE INTO A CANDLE STAND). El verbo hoenge 'colgar' conforma el cuarto cluster que describe eventos de colgar como HANG ROPE OVER TREE BRANCH Y PUT POSTER ON WALL. Quizás en este último caso es interesante mencionar que el danés al utilizar el verbo hoenge 'colgar' parece dar prominencia al hecho de poner (o colgar) algo 
sobre una superficie vertical mientras que esta misma escena en español se utiliza más un verbo que da más prominencia al objeto que permite que la Figura se una a la Base, es decir, el pegamento y por eso, el verbo que se utiliza en esta escena es pegar y no se admitiría colgar ya que requeriría un gancho u otro objeto de donde se pueda colgar la Figura. Este requerimiento del gancho no está presente en la semántica del verbo hoenge 'colgar'. El quinto cluster recoge escenas en las que hay un objeto que se tira o bien a una superficie o bien a un contenedor y el verbo que se utiliza es smide 'tirar'. El verbo stikke 'pegar', 'meter', 'picar', 'punzar', 'pinchar', corresponde al sexto cluster en el que se describen escenas en las que hay una relación de contenedor entre la Figura y la Base. El séptimo cluster se organiza alrededor del verbo putte 'poner, meter' que también describe escenas de contenedor. El octavo cluster se describe principalmente con el verbo tage 'tomar, coger' y recoge escenas de poner y quitar ropa. Finalmente, hay un último cluster, marcado con la línea de puntos discontinua, en la que cada escena se describe predominantemente con un verbo diferente: la escena POUR LIQUID INTO CONTAINER se describe con el verbo hoelde 'verter', la escena SPILL WATER ONTO TABLE con el verbo spilde 'derramar, verter' y la escena GIVE A CUP TO SOMEONE con el verbo give 'dar'.

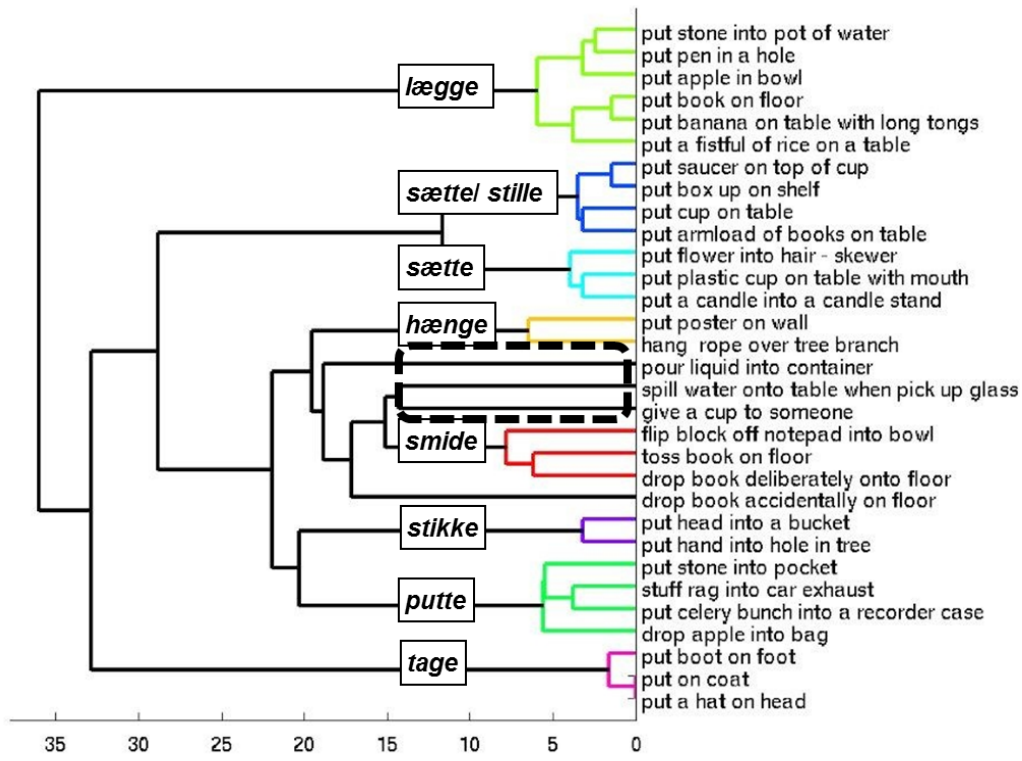

Figura 5: Dendograma para L1 danés 


\section{Discusión: ¿cuántas categorías semánticas hay en español y en danés?}

Los datos sobre la descripción de los eventos de colocación en español y danés que acabamos de revisar en la sección anterior ponen de manifiesto que los hablantes de estas dos lenguas coinciden en algunos casos pero que en la mayoría las categorías semánticas se distribuyen de diferente forma.

En las dos lenguas, ${ }^{2}$ los hablantes coinciden en crear una única categoría que recoge todos los eventos relacionados con la ropa.

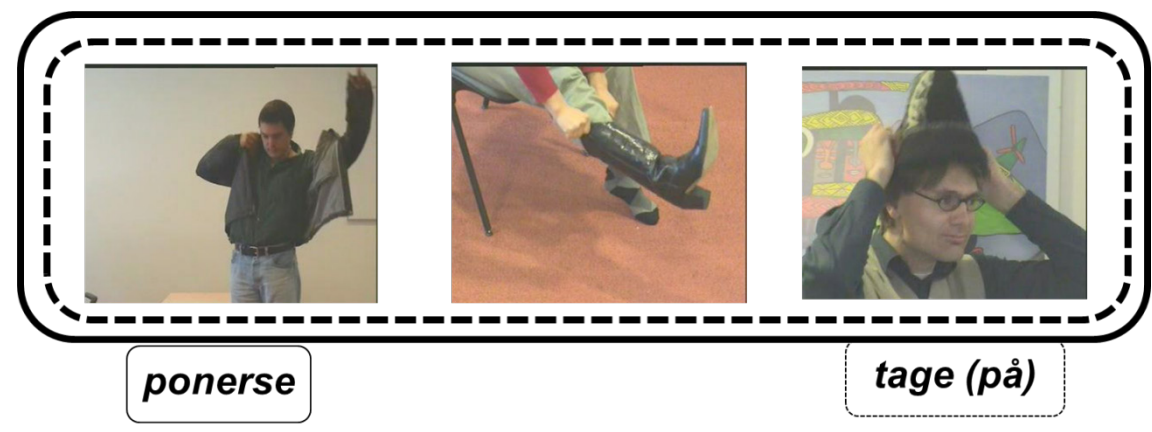

Figura 6: Misma categoría en español y en danés:

Escenas de ropa (BOWERMAN et al. 2004).

Tanto en español como en danés, escenas como PUT BOOT ON FOOT, PUT ON COAT y PUT A HAT ON HEAD que representamos en la Figura 6 se consideran una categoría aparte y ambas lenguas tienen unas estructuras lingüísticas específicas para describirlas: el verbo poner seguido obligatoriamente del se en español y el verbo tage 'tomar, coger' seguido obligatoriamente de la partícula $p a ̊$ 'sobre, encima de' en danés.

Sin embargo, en los demás casos las categorías semánticas no coinciden. Por ejemplo, en los casos en los que se da una relación de apoyo o soporte entre la Figura y la Base como en las escenas que se incluyen en la Figura 7, los hablantes de español las categorizan en el mismo grupo y tienden a describir todos estos casos con el verbo dejar. Los hablantes de danés, por el contrario, dividen el espacio en dos grupos que se describen por medio de los

2 Para que las semejanzas y las diferencias entre las categorías semánticas del español y del danés queden visualmente más claras en las figuras vamos a utilizar la línea continua para las categorías del español y la línea discontinua para las categorías del danés. 
denominados verbos posicionales que se utilizan dependiendo de la orientación y la dimensionalidad espacial. Uno de los grupos utiliza el verbo lagge 'poner / meter horizontalmente', este verbo se usa cuando la Figura tiene una extensión horizontal con respecto a la Base (o cuando la dimensión horizontal del objeto que se coloca es la que predomina) o cuando la Figura carece de una base funcional (SERRA BORNETO, 1996; COVENTRY; GARROD, 2004; GULLBERG; BURENHULT, 2012). El otro grupo se describe con los verbos scette 'poner, meter verticalmente' y stille 'poner, colocar verticalmente' que se utilizan cuando la Figura tiene una extensión vertical con respecto a la Base (o cuando la dimensión vertical del objeto que se coloca es la que predomina) o cuando la Figura tiene una base funcional. En danés, al igual que ocurre en sueco (véase GULLBERG; BURENHULT, 2012) estos tres verbos solamente se utilizan en aquellos casos en los que se ejerce un control manual sobre la Figura hasta que esta llega a la situación de reposo.

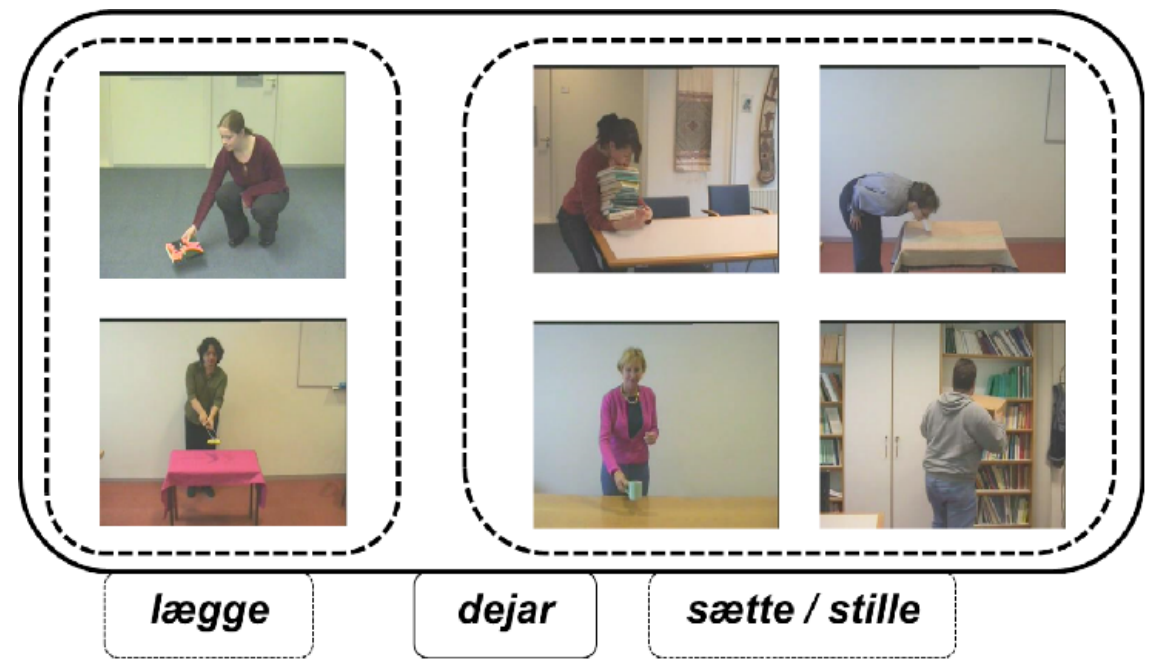

Figura 7: Categorías diferentes en español y danés: Apoyo (BOWERMAN et al. 2004).

Otro ejemplo en el que las categorías no coinciden es el caso de las relaciones de contenedor en el que la Figura se mete (total o parcialmente) en la Base como en las escenas que se incluyen en la Figura 8. 


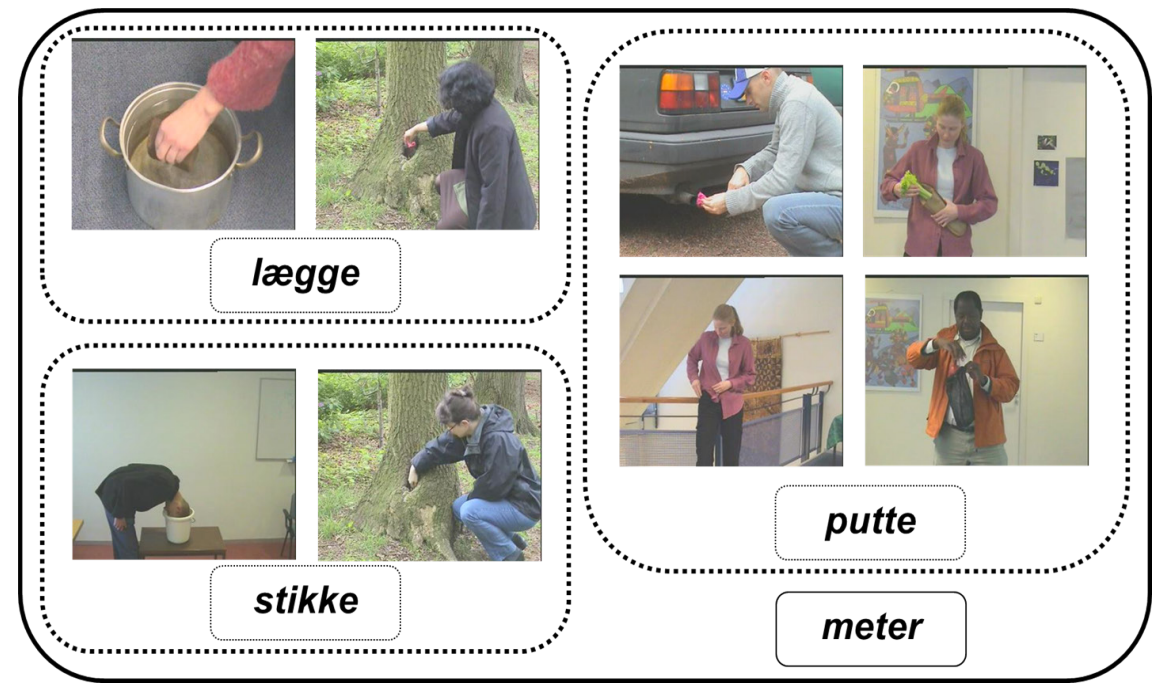

Figura 8: Categorías diferentes en español y danés:

Contenedor (BOWERMAN et al. 2004).

En estos casos, los hablantes de español describen todas estas escenas de contenedor con un único verbo meter mientras que los hablantes de danés forman tres grupos diferentes. El primer grupo utiliza el verbo lagge 'poner / meter horizontalmente' y se utiliza para aquellas escenas en las que la dimensión horizontal de la Figura es la dominante (PUT STONE INTO POT OF WATER, PUT PEN IN A HOLE). Como ya hemos visto en el caso anterior, este mismo verbo lagge se usa tanto para situaciones donde hay una relación de soporte como para situaciones en las que se da una relación de contenedor, así que podemos concluir que la característica que distingue a este verbo es la horizontalidad. El segundo grupo utiliza el verbo stikke 'meter, picar, punzar, pinchar'. Parece ser que este verbo se usa en escenas en las que el contenedor, la Base, tiene una cierta profundidad de tal modo que la Figura, que suele ser puntiaguda (p. ej. una aguja) parece seguir la trayectoria paralela a la de la Base (PUT hAND into hole IN TREE). También es importante destacar que cuando se utiliza este verbo se da una relación de encaje ajustado entre la Figura y la Base (PUT HEAD INTO A BUCKET). El tercer grupo utiliza el verbo putte 'poner, meter' que parece ser el menos específico de todos. Este verbo se suele usar normalmente en discurso oral y cubre acciones diferentes 
como el encaje ajustado (STUFF RAG INTO CAR EXHAUST, PUT CELERY BUNCH INTO A RECORDER CASE) pero también el encaje holgado (DROP APPLE INTO BAG), por ello, podemos concluir tentativamente que el verbo putte se utiliza para casos en los que hay una relación en que la Figura está protegida por la Base, en los que la Figura pasa a ser suavemente una parte integrada de la Base contenedora. A juzgar por estos datos parece que entre los verbos daneses lagge-stikke-putte existen algunas diferencias sutiles; sin embargo, los datos que hemos recogido a través de estos videos no son suficientes para establecer exactamente cómo se diferencian estos verbos. Por ello, futuras investigaciones deberán de centrarse en estos aspectos e intentar a través de estudios tanto experimentales como de corpus describir el uso de estos verbos daneses y las diferencias semánticas que existen entre ellos.

Finalmente, otro de los ejemplos en los que las categorías semánticas en las dos lenguas no coinciden se encuentra en la conceptualización de dos características del evento de colocación: la intencionalidad, es decir, el grado de participación del Agente y la dinámica de la fuerza, es decir, la forma en la que el Agente y la Figura interactúan con respecto a la fuerza que se emplea para llevar a cabo el evento (veáse IBARRETXE-ANTUÑANO, 2012; TALMY, 1988). La intencionalidad y la dinámica de la fuerza interactúan entre sí y por eso, es posible distinguir entre diferentes casos de eventos de colocación. En los estímulos hay tres escenas que representan tres de las posibles situaciones en las que se unen estos dos elementos para describir diferentes tipos de escenas de colocación. La primera escena, DROP BOOK ACCIDENTALLY ON FLOOR, describe a un hombre que entra andando con una pila de libros en sus brazos y se le cae uno sin querer. En la segunda escena, DROP BOOK DELIBERATELY ONTO FLOOR, se ve a una mujer sentada en una silla con un libro en la mano al que deja caer. En la tercera escena, TOSS вОок ON FLOOR, hay un hombre sentado en una silla que lanza el libro que tiene en su mano al suelo. 


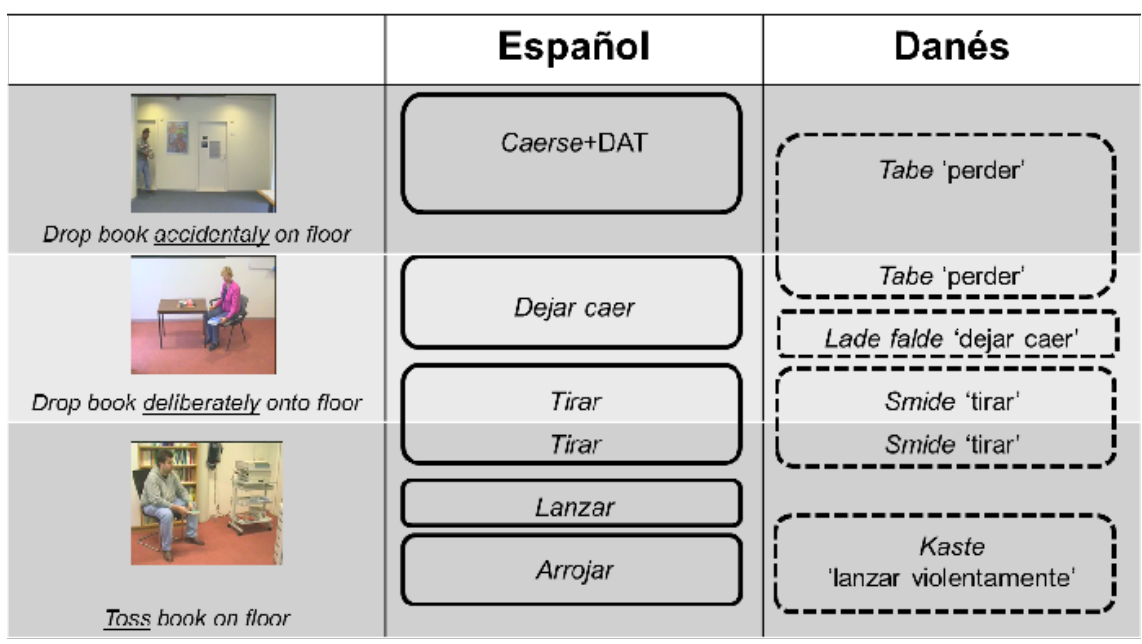

Figura 9: Categorías diferentes en español y danés:

intencionalidad y dinámica de la fuerza (BOWERMAN et al. 2004).

Como se puede apreciar en la Figura 9, los hablantes de español y danés difieren en el número de categorías semánticas. Los españoles utilizan cinco construcciones diferentes en las que se observa una progresión en cuanto a la intencionalidad y la dinámica de la fuerza; para la primera escena se utiliza la construcción caerse+dativo que implica una participación inconsciente del agente que no tiene ninguna intencionalidad de tirar el libro al suelo y por lo tanto, la dinámica de la fuerza también es baja. En la segunda escena, se utilizan dos construcciones diferentes. La construcción dejar caer señala que el agente participa en el evento pero con poca dinámica de fuerza y la construcción con el verbo tirar implica una mayor fuerza por parte del agente. Finalmente, en la escena tercera se utilizan tres construcciones diferentes. En todos los casos los verbos que se emplean indican la participación del agente pero con grados de fuerza cada vez mayores según esta escala: tirar < lanzar $<$ arrojar. Los daneses, por otro lado, utilizan solamente cuatro construcciones. La construcción con el verbo tabe 'perder, dejar caer' que se utiliza en las dos primeras escenas, aunque es posible que su uso en la segunda escena se deba más al hecho de que los hablantes hayan interpretado la situación como que el Agente tira el libro 'sin cuidado';3

3 Queremos dar las gracias a Jørgen Bang por esta observación. 
la construcción con el verbo lade falde 'dejar caer' para la segunda escena, la construcción con el verbo smide 'tirar' para las escenas segunda y tercera que el Agente y la construcción con el verbo kaste 'lanzar violentamente' para la última escena. Es decir, los hablantes de danés también distinguen diferentes grados de intencionalidad y dinámica de la fuerza, pero dividen el espacio semántico en menos categorías que los hablantes españoles.

\section{Conclusiones: ¿qué tiene que hacer el aprendiente danés de español como L2?}

En las páginas anteriores hemos hecho un repaso de cómo los hablantes nativos de español y danés conceptualizan y describen los eventos de colocación en sus respectivas lenguas. Hemos visto que en algunos casos, las categorías semánticas en las que se dividen este tipo de eventos son coincidentes - por ejemplo, en las escenas relacionadas con la ropa en las que el danés y el español utilizan una única construcción especial para describir estos eventos. Sin embargo, también hemos mostrado cómo hay varios casos en los que las categorías semánticas no coinciden, bien porque el danés divide el espacio semántico en más categorías debido a la necesidad de describir entre otros aspectos la posición vertical u horizontal de la Figura (por ejemplo, los casos de apoyo y contenedor), bien porque el español hace una distinción más sutil de las relaciones entre la intencionalidad y la dinámica de la fuerza.

Estas diferencias son cruciales si nos ponemos en la perspectiva del aprendiente de segundas lenguas, ya que las categorías semánticas entre las dos lenguas que maneja no son las mismas. En el caso de los eventos de apoyo y de contenedor, los aprendientes tienen que cambiar de un número mayor de categorías en su lengua nativa danesa a una sola categoría en español L2. Para los casos de apoyo, el aprendiente danés tiene la distinción entre laegge y scette/stille que tiene que adaptarla a la única categoría del verbo dejar. Para los casos de contenedor, el aprendiente danés tiene que pasar del trío loegge, stikke y putte a una única opción en español con el verbo meter. En relación con la intencionalidad y la dinámica de fuerzas, los aprendientes tienen que realizar la operación contraria. Es decir, tienen que cambiar de un número menor de categorías en el danés L1 a un mayor número de categorías en el español L2: tabe correspondería a las construcciones españolas, 
caerse+dativo y dejar caer, lade falde a dejar caer, smide al verbo tirar, y kaste a los verbos lanzar y arrojar. El hecho de que las categorías semánticas entre las dos lenguas no coincidan constituye una de las dificultades más importantes para un aprendiente danés de español como L2 por varias razones. Por un lado, hay que tener en cuenta que el aprendiente tiene que adaptar las categorías de su lengua materna (reduciéndolas o aumentándolas) a las de la segunda lengua, y por otro lado, es importante destacar que el aprendiente además tiene que conocer los elementos que aparecen en estas construcciones tales como el uso del clítico se en español o verbos bastante específicos como lanzar frente a verbos más generales y comunes como poner. Aunque es una cuestión abierta a la espera de los datos empíricos con aprendientes daneses de español como L2, nuestra hipótesis postula que, dependiendo del nivel de los aprendientes, estos se encontrarán con dificultades a la hora de codificar estos eventos de colocación especialmente con respecto a estos dos puntos: falta de coincidencia entre categorías semánticas y falta de conocimiento de estructuras específicas. Por supuesto, desde un punto de vista estrictamente gramatical, el hecho de que un aprendiente utilizara un verbo general como poner en alguna de estas escenas sería absolutamente correcto. Por ejemplo, en una escena de contenedor como PUT PEN IN A HOLE, un enunciado como la mujer pone el rotulador en el árbol no es incorrecto desde el punto de vista gramatical. Sin embargo, como se comentó en la sección 2, uno de los aspectos fundamentales de la teoría del pensar para hablar de Slobin se basa precisamente en señalar que cada lengua tiene su propio estilo retórico, es decir, que cada lengua, o mejor dicho, hablante, utiliza una serie de expresiones y construcciones para determinados casos. Estas expresiones no son requerimientos gramaticales de obligado cumplimiento, sino tendencias discursivas que los hablantes nativos muestran a la hora de describir una determinada situación. Por ello, además de las dos dificultades que acabamos de señalar, un aprendiente se enfrenta también a la dificultad que supone la adaptación del estilo retórico de su lengua materna al de su segunda lengua, o como algunos autores han denominado a un aprender a pensar para hablar diferente o a repensar para hablar (CADIERNO, 2004, 2010; ROBINSON; ELLIS, 2008). 


\title{
The expression of placement events in Danish and Spanish
}

\begin{abstract}
Placement events, that is, situations where a Figure changes its position by the action of an agent (e.g., Pone una taza en la mesa '(He/she) puts a cup on the table'), constitute an interesting research area within Cognitive Linguistics, especially in research related to neorelativistic issues. This study first investigates the linguistic codification of placement in two typologically different languages (TALMY, 1991, 2000): Danish (satelliteframed language, CADIERNO, 2004) and Spanish (verb-framed language, TALMY, 1991; SEBASTIÁN and SLOBIN, 1994). Secondly, and on the basis of the findings obtained in the crosslinguistic description, we formulate, from the perspective of thinking for speaking (CADIERNO, 2008; SLOBIN 1991), a series of hypotheses about the strategies and difficulties that Danish speaking learners of L2 Spanish may encounter when talking about this type of event. Data were elicited by means of the stimuli developed by the PUT-project at the Max Planck Institute for Psycholinguistics in Nijmegen (The Netherlands) (BOWERMAN et al., 2004, KOPECKA; NARASIMHAN, 2012).
\end{abstract}

Keywords: Placement events. Spanish. Danish. Intertypological variation. Thinking for speaking.

Referências

BOWERMAN, Melissa; GULlBERG, Marianne; MAJID, Asifa; NARASHIMHAN, Bhuvana. Put project: The cross-linguistic encoding of placement verbs. In: MAJID, Asifa. Field Manual 9. Max Planck Institute for Psycholinguistics. Language and cognition group. Nimega: MPI, 2004.

CADIERNO, Teresa. Expressing motion events in a second language: A cognitive typological perspective. In: ACHARD, Michel; NIEMEIER, Susanne. Cognitive linguistics, second language acquisition, and foreign language teaching. Berlín: Mouton de Gruyter, 2004. p. 13-49.

CADIERNO, Teresa. Learning to talk about motion in a foreign language. In: ROBINSON, Peter; ELLIS, Nick C. Handbook of cognitive linguistics and second language acquisition. New York y London: Routledge, 2008. p. 239-275. 
CADIERNO, Teresa. Motion in Danish as a second language: Does the learner's L1 make a difference? In: HAN, ZhaoHong; CADIERNO, Teresa. Linguistic relativity in SLA: Thinking for speaking. Bristol: Multilingual Matters, 2010. p. 1-33.

COVENTRY, Kenny R.; GARROD, Simon C. Saying, seeing and acting: The Psychological semantics of spatial prepositions. Essays in Cognitive Psychology Series. Hove y New York: Psychology Press, 2004.

GULlBERG, Marianne; BURENHULT, Niclas. Probing the linguistic encoding of placement and removal events in Swedish. In: KOPECKA, Anetta; NARASINMHAN, Bhuvana. Events of 'putting' and 'taking': A crosslinguistic perspective. Amsterdam y Filadelfia: John Benjamins, 2012. p. 167-182.

KOPECKA, Anetta; NARASIMHAN, Bhuvana (Ed.). The events of 'putting' and 'taking': A crosslinguistic perspective. Amsterdam y Filadelfia: John Benjamins, 2012.

IBARRETXE-ANTUÑANO, Iraide. Placement and removal events in Basque and Spanish. In: KOPECKA, Anetta; NARASIMHAN, Bhuvana. Events of 'putting' and 'taking': A crosslinguistic perspective. Amsterdam y Filadelfia: John Benjamins, 2012. p. 123-143.

MAJID, Asifa. A guide to stimulus-based elicitation for semantic categories. In: THIEBERGER, Nicholas. The Oxford handbook of linguistic fieldwork. New York: Oxford University Press, 2012. p. 54-71.

MAJID, Asifa; BOWERMAN, Melissa; VAN STADEN, Miriam; BOSTER, James S. The semantic categories of cutting and breaking events. A crosslinguistic perspective. Cognitive Linguistics, v. 18, n. 2, p. 133-152, 2007.

NARASIMHAN, Bhuvana; KOPECKA, Anetta; BOWERMAN, Melissa; GULLBERG, Marianne; MAJID, Asifa. Putting and taking events. A crosslinguistic perspective. In: KOPECKA, Anetta; NARASIMHAN, Bhuvana. Events of putting and taking: a crosslinguistic perspective. Amsterdam y Filadelfia: John Benjamins, 2012. p. 1-18.

ROBINSON, Peter; ELLIS, Nick C. Handbook of cognitive linguistics and second language acquisition. New York y London: Routledge, 2008.

SEBASTIÁN, Eugenia; SLOBIN, Dan Isaac. Development of linguistic forms: Spanish. In: BERMAN, Ruth Aronson; SLOBIN, Dan Isaac. Relating events in narrative: A cross-linguistic developmental study. Hillsdale, N.J.; Lawrence Erlbaum, 1994. p. 239-284. 
SERRA BORNETO, Carlo. Liegen and stehen in German. A study in horizontality and verticality. In: CASAD, Eugene $H$. (Ed.). Cognitive linguistics in the redwoods. Berlín: Mouton de Gruyter, 1996. p. 459-505.

SLOBIN, Dan Isaac. Learning to think for speaking: Native language, cognition, cognition, and rhetorical style. Pragmatics, v. 1, p. 7-26, 1991.

SLOBIN, Dan Isaac. Two ways to travel: Verbs of motion in English and Spanish. In SHIBATANI, Masayoshi; THOMPSON, Sandra A. Grammatical Constructions: Their Form and Meaning. Oxford: Clarendon Press, 1996. p. 195317.

SLOBIN, Dan Isaac. Verbalized events: A dynamic approach to linguistic relativity and determinism. In NIEMEIER, Susanne; DIRVEN, René (Ed.). Evidence for linguistic relativity. Amsterdam y Philadelphia: John Benjamins, 2000. p. 107138.

SLOBIN, Dan Isaac. The many ways to search for a frog. In: STRÖMQVIST, Sven; VERHOEVEN, Ludo (Ed.). Relating Events in Narrative: Typological and Contextual Perspectives. Hillsdale, NJ: Lawrence Erlbaum Associates, 2004. p. 219-257.

SLOBIN, Dan Isaac. What makes manner of motion salient? Explorations in linguistic typology, discourse, and cognition. In: HICKMAN, Maya; ROBERT, Stéphane. Space in languages: Linguistic systems and cognitive categories. Amsterdam y Philadelphia: John Benjamins, 2006. p. 59-81.

TALMY, Leonard. Lexicalization patterns: Semantic structure in lexical forms. In: SHOPEN, Timothy (Ed.). Language typology and syntactic description: Grammatical categories and the lexicon. Cambridge: Cambridge University Press, 1985. p. 36-149.

TALMY, Leonard. Force dynamics in language and cognition. Cognitive Science, v. 12, p. 49-100, 1988.

TALMY, Leonard. Path to realization: A typology of event conflation. En: Proceedings of the seventeenth annual meeting of the Berkeley Linguistic Society, v. 17 , p. $480-519,1991$

TALMY, Leonard. Towards a cognitive semantics. Cambridge, MA: The MIT Press, 2000. 